\title{
An experimental study of girder-borne sound pressure emission and acceleration
}

\author{
Lukasz Szyszka \\ Department of Structural Mechanics; Faculty of Civil and Environmental Engineering \\ and Architecture; Rzeszow University of Technology; \\ Poznańska St. 2, 35-084 Rzeszow, Poland; \\ lszyszka@prz.edu.pl (DD 0000-0001-5017-7611
}

\begin{abstract}
The subject of this paper is an experimental study on girder-borne sound pressure emission. The sources of the excitation are a hammer and a modal shaker. The structure researched is a simply supported steel plate girder of the size: $7.95 \mathrm{~m} \mathrm{x} 1.5 \mathrm{~m}$. The study covers the acceleration and sound pressure level measurements (SPL), and SPL estimation based on the accelerations measured. The correlation factor for higher estimation accuracy in lower frequency bands is proposed.
\end{abstract}

Keywords: SPL, vibroacoustics, modal analysis, acceleration, noise

\section{Introduction}

In Poland, there are over 1000 railway bridges with a steel supporting structure [1]. A rapid expansion of modern Computer Aided Design techniques in civil engineering results in designing taller, slimmer, longer and lighter structures. It also leads to the problem of noise generation. Considering noise pollution as a hindrance for the people living nearby, multiple fields of research were created, where the objective is to prevent the excessively high levels of noise generated by the everyday exploitation and self- generated vibrations of the structure. An estimation of the sound pressure level values for the newly designed structures has become an important task for structural designers and constructors. An analytical approach to the sound pressure calculation allows to estimate the noise generated by the structure designed, however due to the fact that analytical models characterise with simple subsystems (e.g. plate, bar), they do not describe complex structures with good accuracy, or are time consuming and extremely hard, if at all possible, to create. This fact works in favour of computer methods. There are numerous works where Finite Element Method (FEM) [2-4], Boundary Element Method (BEM) [5], Statystical Energy Analysis (SEA) [6], or a combination of the two aforementioned methods [7-9], were used for calculation of bridge vibration and sound pressure emission. The task of calculating higher frequency of complex structures needs smaller FEM 
elements, which makes the task heavier on computing and is not practical [10]. This results in the need of simpler noise estimation methods that could be used at the stage of designing a newly built structure.

\section{Experimental study of girder-borne sound pressure level and acceleration}

\subsection{An outline of the test subject}

The test subject is a welded steel plate girder that has $0.4 \mathrm{~m}$ wide flanges and $1.5 \mathrm{~m}$ high web. The thickness of the web and flange are $12 \mathrm{~mm}$ and $16 \mathrm{~mm}$, respectively. An effective span of the beam in the axis of the supports is equal to $7.2 \mathrm{~m}$ with a total beam length of $7.95 \mathrm{~m}$. The girder is designed as a plate girder, with additional braces, as stated in Fig 1. All elements of the beam are made of steel type S355JR, the density of the steel is assumed as $\rho=7850 \mathrm{~kg} / \mathrm{m} 3, \mathrm{E}=210 \mathrm{GPa}$.

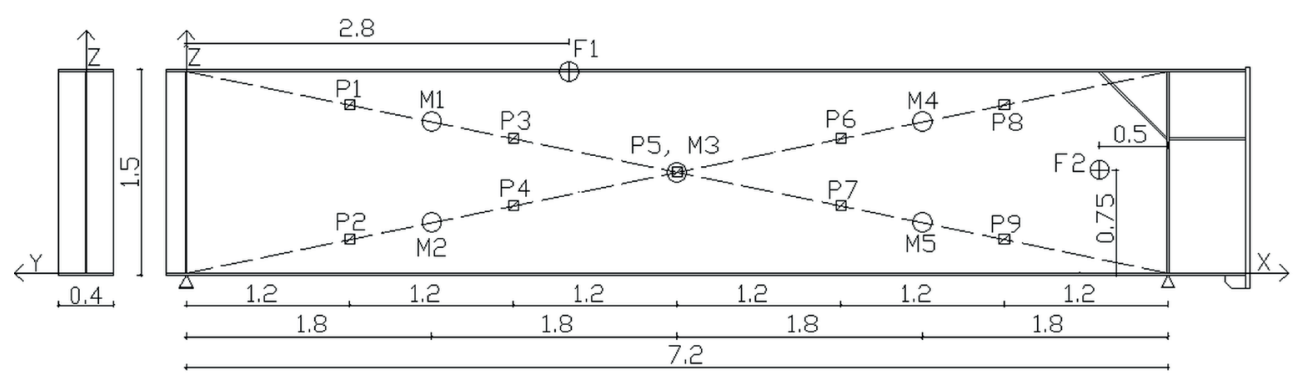

Fig. 1. A cross section and static scheme of the beam with the location of the sensors. M1...M5 and P1... P9 stand for the locations of microphones and accelerometers, respectively, F1 and F2 are locations of force excitation. Distance values are expressed in meters

The structure tested is a simply supported beam, subjected to a dead load, which can represent a bearing part of the bridge structure, or a compound of such structure. The tests were made in Departmental Laboratory of Construction Research at the Faculty of Civil Engineering, Environmental Engineering and Architecture, at Rzeszow University of Technology. The subject of the experimental research was a steel plate girder beam. Dimensions of the beam were pointed out in Fig. 1. The simply supported beam scheme was assumed. Sound pressure measurements were taken in a frequency band $20 \mathrm{~Hz}-5000 \mathrm{~Hz}$. A total of 9 accelerometers were located directly at the surface of the plate, further 5 acoustic microphones were located at a $10 \mathrm{~cm}$ distance from the object researched, and the locations of the specific sensors were featured in Fig. 1. The grid of sensors was dictated by the plan of further numerical data analysis that involved generation of shapes and its comparison, and FEM model validation, but only a part of all measurements was taken into account in this paper. It was also needed to average SPL measurements.

\subsection{A research method description}

The purpose of the research was to compare the results obtained from performing the analytical calculation based on the acceleration measurements, to estimate and confront it with 
SPL readings obtained from sound pressure measurements. In research, one of the experimental modal analysis methods - the SIMO (Single Input - Multiple Output) method was used. In the SIMO method with an application of the electrodynamic modal shaker and modal hammer interchangeably as a generator of the input signal (Single Input) the possibility for simultaneous measurement in many measuring points (Multiple Output) located on the test subject was provided.

\subsection{Force excitation}
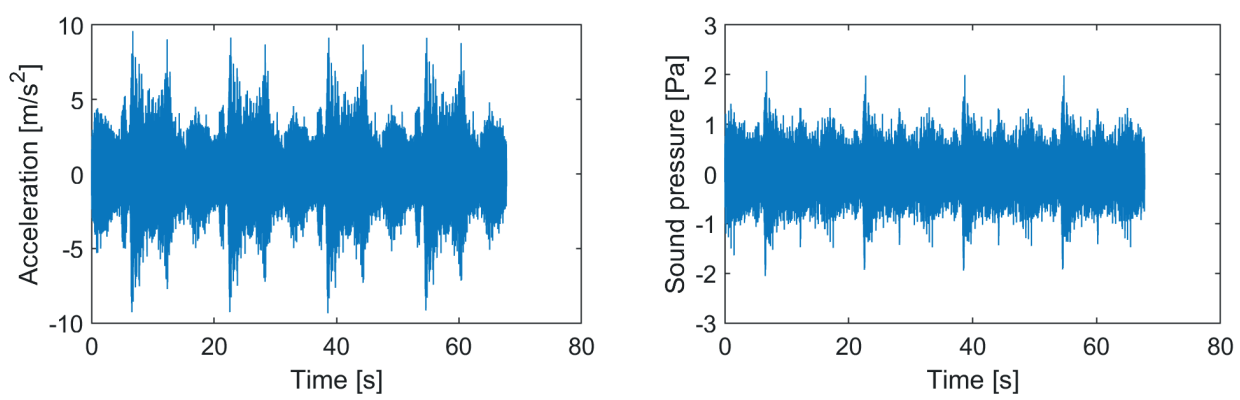

Fig. 2. Exemplary measured signal for sensor P5 and M3, respectively for shaker excited run
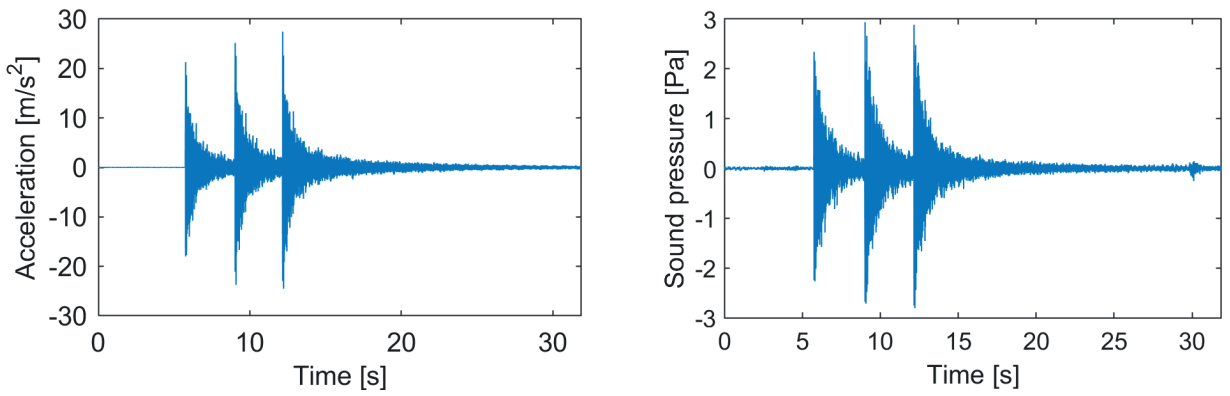

Fig. 3. Exemplary measured signal for sensor P5 and M3, respectively for impact excited run

If An excitation method of the time-varying force was provided with the use of an electrodynamic modal shaker. The modal shaker was made by ModalShop, model 2100E11. The modal shaker was attached to a testing stand by suspending it with 4-steel wires to rigid column with a fixed hanger. The shaker was controlled by the third-party software and hardware, independent from measuring devices to simulate a free run scenario. The excitation point was located on a top flange in point F1 (Fig. 1). The shaker excitation (Fig. 2) was applied with a sinusoidal and chirp type of excitation with different settings. Excitation direction was applied in horizontal direction Y, by a tension bar. The string on one end was connected by a threaded nut, glued to the top flange of the girder perpendicularly to the longitudinal axis $\mathrm{X}$ of the beam, and on the other end was connected by a clamp with shakers armature.

The second type of force excitation was the impact excitation, where a modal hammer was used. The modal hammer was used as a regular impulse excitation with soft, plastic, head cover, without a force measuring sensor. The impulse signal (Fig. 3) was generated by applying 3 consecutive hits with approximately 1 second intervals of the hammers head in 
horizontal direction $\mathrm{Y}$, perpendicularly to the longitudinal axis $\mathrm{X}$ in point $\mathrm{F} 2$ located at the web of the girder (Fig. 1).

\subsection{Measurement of the response}

Vibrations of the beam were measured by simultaneous registration of the acceleration response in 9 points. Measuring points, where piezoelectric accelerometers were located on the diagonal of the rectangle, as shown in Fig. 1. The sensors applied were 1D B\&K (Bruel\&Kaer, type 4507 B006), piezoelectric accelerometers. The sensors were placed in the horizontal direction $\mathrm{Y}$, perpendicularly to the longitudinal axis $\mathrm{X}$, in the same direction as the excitation was applied.

Sound pressure levels were measured by microphones. The sensors used were 1D B\&K, type 4961. Microphones were located with similar pattern as accelerometers with a slightly different grid (Fig. 1). The sensors were placed in the horizontal direction Y, perpendicularly to the longitudinal axis $\mathrm{X}$, in the same direction as the excitations F1 and F2 were applied, located $10 \mathrm{~cm}$ from the plate (Fig. 1).

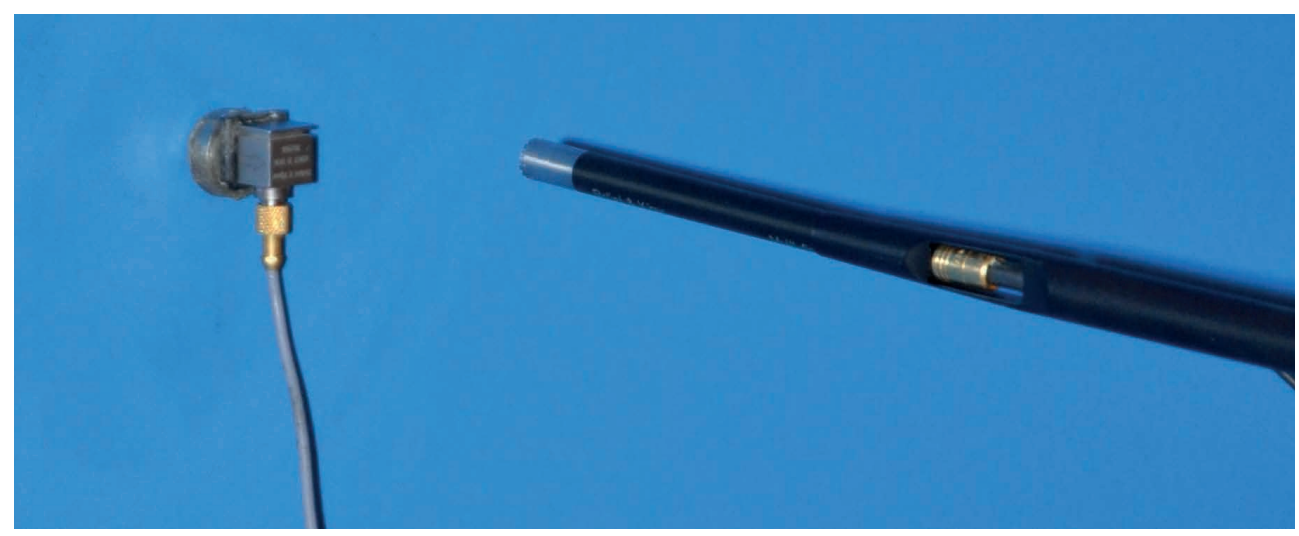

Fig. 4. Photography of M3 and P5

\section{Data Analysis}

\subsection{Calculations}

By using an analytical calculation in order to get a rough estimation of the sound-pressure emission, the structure was simplified to the infinite plate model, and the sound pressure emission and SPL were calculated with the formula (1) and (2) respectively:

$$
p(x, y)=\frac{v_{0} \cdot \rho \cdot c}{\sqrt{1-k_{B}^{2} / k^{2}}} \cdot e^{-j \cdot k_{B} \cdot x} \cdot e^{-j \sqrt{k^{2}-k_{B}^{2}} \cdot y}
$$

Tables where:

$v_{0}$ - mean square velocity of vibrating plate

$\rho$ - density of the ambient medium

$c$ - propagation velocity of the longitudinal wave in ambient medium 
$k_{\mathrm{B}}$ - frequency dependant wavenumber of the plate vibrations

$k$ - frequency dependant wavenumber of the surrounding medium

$\mathrm{j}=\sqrt{-1}$

$x$ - distance measured along the length of the plate

$y$ - distance measured perpendicularly to the surface of the plate

$\mathrm{SPL}=10 \log _{10}\left(\frac{p(x, y)^{2}}{p_{0}}\right)$

(2) $[11$, eq. (3)]

where $p_{0}$ is reference value for pressure and $p_{0}=2 \cdot 10^{-5} \mathrm{~N} / \mathrm{m}^{2}$

In order to calculate the averaged sound pressure emission from the beam, the formula (1) used for calculating infinite plate generated pressure was adopted. The pressure generated by the plate was calculated with the formula (1) for points M3 and P5 with consideration to their position, they were located in the intersection of the diagonals of the before mentioned rectangular plate (Fig. 1). $V_{0}$ value was obtained from the integration of the accelerometer's measurements for $1 / 3$ octave bands, and pressure was also calculated for the respective frequency bands [11]. Based on the results of the above calculations of pressure, the respective SPLs were computed with the formula (2).

\subsection{Results of measurements}

In order to achieve higher correlation between measured and calculated data, frequency dependant, dimensionless, correlation factor lc based on wavelength number of sound propagation in air was adopted into (1) resulting with the formula (3).

$p_{c}(x, y)=\frac{1}{\lambda_{c}} \frac{v_{0} \cdot \rho \cdot c}{\sqrt{1-k_{B}^{2} / k^{2}}} \cdot e^{-j \cdot k_{B} \cdot x} \cdot e^{-j \sqrt{k^{2}-k_{B}^{2}} \cdot y}$

where:

$l_{\mathrm{c}}$ - wavelength in ambient medium [-]

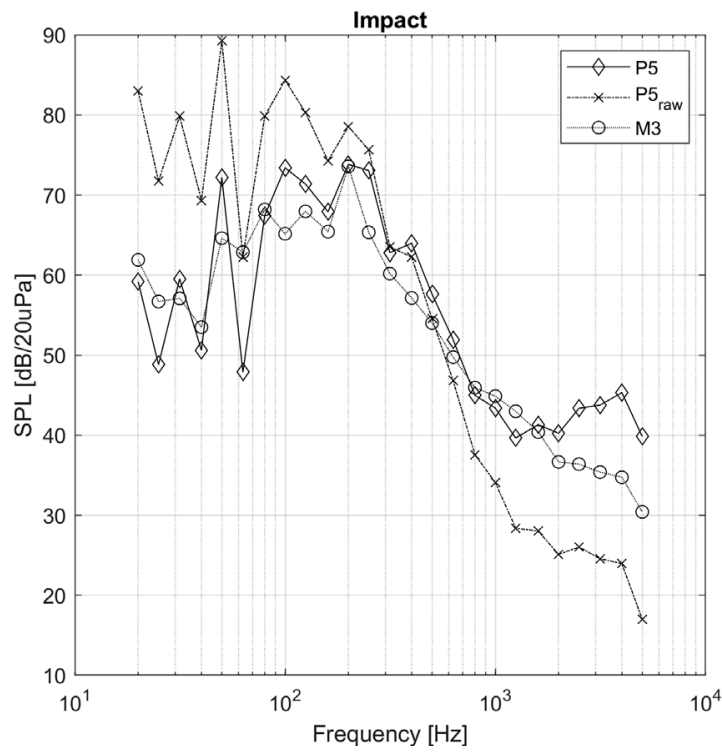

\begin{tabular}{|c|c|c|c|}
\hline$f[\mathrm{~Hz}]$ & $\mathrm{P} 5[\mathrm{~dB}]$ & $\mathrm{P} 5_{\text {raw }}[\mathrm{dB}]$ & $\mathrm{M} 3[\mathrm{~dB}]$ \\
\hline 20 & 59.17 & 82.97 & 61.89 \\
\hline 25 & 48.80 & 71.74 & 56.69 \\
\hline 31.5 & 59.54 & 79.82 & 57.09 \\
\hline 40 & 50.60 & 69.33 & 53.49 \\
\hline 50 & 72.19 & 89.25 & 64.61 \\
\hline 63 & 47.86 & 62.28 & 62.85 \\
\hline 80 & 67.43 & 79.83 & 68.19 \\
\hline 100 & 73.40 & 84.34 & 65.15 \\
\hline 125 & 71.36 & 80.31 & 67.95 \\
\hline 160 & 67.90 & 74.26 & 65.41 \\
\hline 200 & 73.83 & 78.55 & 73.57 \\
\hline 250 & 73.05 & 75.63 & 65.34 \\
\hline 315 & 62.79 & 63.51 & 60.18 \\
\hline 400 & 63.97 & 62.24 & 57.14 \\
\hline 500 & 57.62 & 54.55 & 54.01 \\
\hline 630 & 51.94 & 46.81 & 49.75 \\
\hline 800 & 44.99 & 37.55 & 45.94 \\
\hline 1000 & 43.37 & 34.09 & 44.88 \\
\hline 1250 & 39.64 & 28.38 & 42.98 \\
\hline 1600 & 41.32 & 28.03 & 40.39 \\
\hline 2000 & 40.24 & 25.10 & 36.66 \\
\hline 2500 & 43.34 & 26.03 & 36.38 \\
\hline 3150 & 43.75 & 24.55 & 35.39 \\
\hline 4000 & 45.32 & 23.95 & 34.73 \\
\hline 5000 & 39.83 & 16.99 & 30.41 \\
\hline
\end{tabular}

Fig. 5. Diagrams of SPL in $1 / 3$ octave frequency bands for the impact excitation in selected run. P5 $5_{\text {raw }}-$ SPL calculated with formula (1), P5 - SPL calculated with formula (3), M3- measured SPL used as reference data 


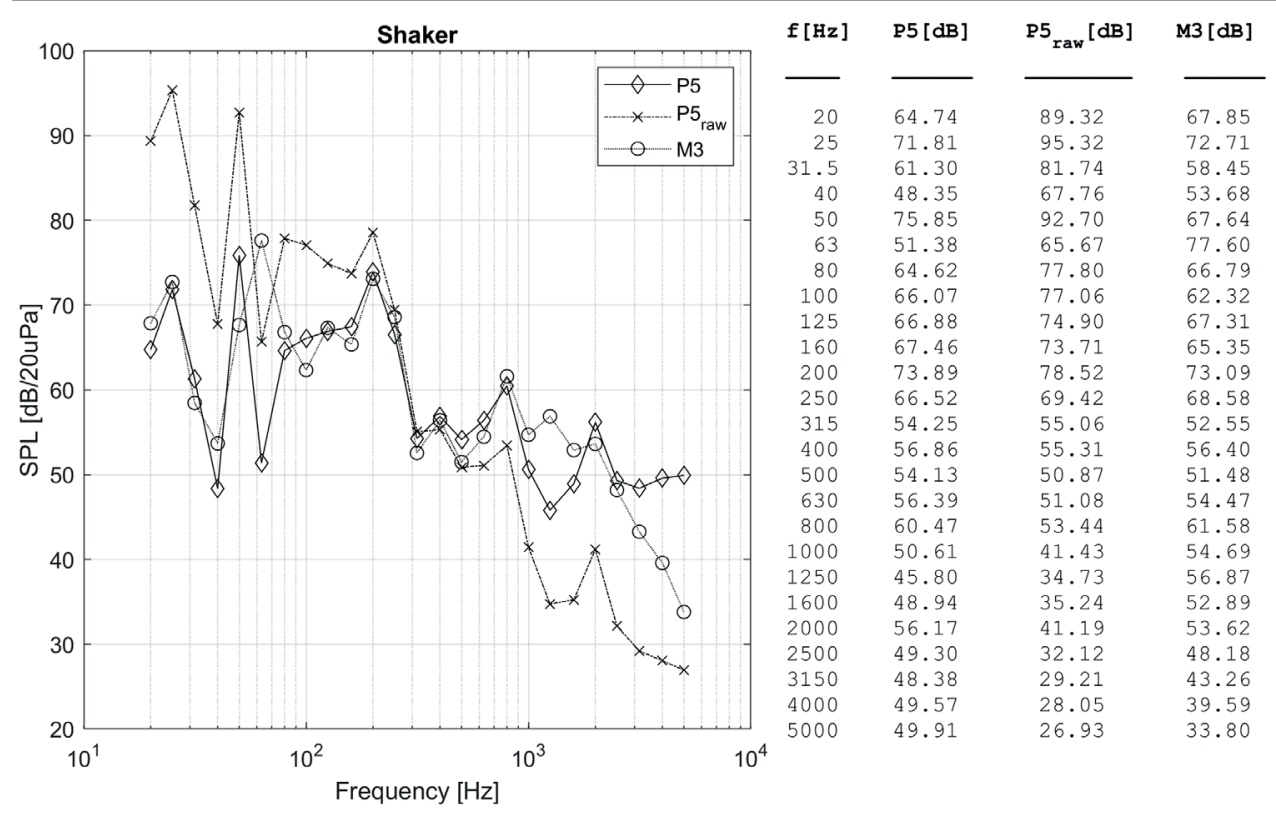

Fig. 6. Diagram of SPL in $1 / 3$ octave frequency bands for the shaker excitation in selected run. P5 $5_{\text {raw }}-$ SPL calculated with formula (1), P5 - SPL calculated with formula (3), M3- measured SPL used as reference data

The SPLs measured and calculated in 1/3-octave centre frequency band are shown in Fig. 5 and Fig. 6 . Total of 6 cases for modal shaker excitation and 4 of the impulse types of the excitation were analysed, and 2 exemplary runs were selected. The calculation using formula (1) was performed to get SPL values $\mathrm{P}_{\text {raw }}$. Considerable differences in results might have come from the free run scenario, and the lack of force measurements, which could have been used to normalize measured data. Due to the poor correlation equalling even up to $25 \mathrm{~dB}$ difference (Fig. 6.), of estimated SPL $\left(\mathrm{P}_{\text {raw }}\right)$ with measurement data (M3) in the whole frequency band, in both cases of excitation, correlation factor dependant on the frequency for formula (1) was proposed. Using this factor allowed to obtain the formula (3) and a better consistence between the estimation and measurement in frequency band of $20-800 \mathrm{~Hz}$ for shaker and $20-2000 \mathrm{~Hz}$ for impact excitation with the exception of $63 \mathrm{~Hz} \mathrm{1/3}$ octave band (Fig. 5). The Root Mean Square Error (RMSE) equalled to 12.92 , and 21.91 respectively. The percentage difference is $41 \%$ between the two.

The dynamic response of the girder is derived from free vibrations of the beam. The dominant frequency of web acceleration and SPL generation is in the range of 80-400 Hz when vibrations are excited with a hammer (Fig. 5). The peak frequency of the web acceleration is at $200 \mathrm{~Hz}$ (Fig. 5), and three types of calculated results.

The second type of measurement vibrations are excited by a modal shaker. The dominant frequency of web acceleration is $20-200 \mathrm{~Hz}$, which is in agreement with the excitation range covering low frequencies up to $256 \mathrm{~Hz}$., with sudden drop to 40-63 Hz, when vibrations are excited with modal shaker (Fig. 6). The peak frequency of the web acceleration is at $63 \mathrm{~Hz}$ (Fig. 6). The plot shows comparison of the referential pressure signal of the microphone M3 (Fig. 1) in 1/3 octave centre frequency band, and two types of calculated results. The RMSE 
in this case equalled to 13.77 , and 23.87 , respectively. That gives the percentage difference at $42 \%$ between the two.

Due to the fact that the measurements were conducted indoors, the background noise was neglected. An experimental modal analysis was made using B\&K PULSE Reflex commercial software. The software was used only for the registration of raw pressure and acceleration signals. Further data analysis was done with the usage of Matlab software. For every data

sample, the respective FRF (Frequency Response Function) were computed [12].

The measurement sampling frequency of $16384 \mathrm{~Hz}$ was used to obtain FRF functions in the range of $20-5000 \mathrm{~Hz}$, for both acceleration and pressure measurements, with the frequency resolution of $0.0625 \mathrm{~Hz}(1 / 16 \mathrm{~Hz})$. Digital filtering and computation were performed in order to obtain results in $1 / 3$ octave frequency bands.

Exemplary results for the modal shaker excitation are shown in Fig. 2, while Fig. 3 shows the results for impulse excitation. The analysed part of the impact signal started from the first hit to the end of the signal and its free vibrations. The first part of the signal containing only signal and measurement noise got removed from the analysis.

\section{Conclusions}

The purpose of the research was to compare the results obtained from performing an analytical calculation based on velocity, which is obtained by integrating an acceleration signal to estimate and compare it with referential values, based on the measured sound pressure level. Based on the vibration analysis, a prediction approach of the steel girder was presented and applied to the noise analysis of a simply supported beam. The field test was carried out to verify the analytical estimation. The following remarks can be made.

Considering the impact excitation, the analytically calculated SPLs with the use of the correlation factor corresponds to the girder-borne noise in good agreement in the range of 20-1000 Hz. There is an increasing difference between the values with consideration of the proposed factor, especially in the higher frequency range of $630-5000 \mathrm{~Hz} 1 / 3$ octave bands when using raw $\mathrm{P} 5$ signal.

For analytically calculated SPLs corresponding to girder-borne noise, resulting from modal shaker excitation, the values P5 and M3 are also compatible in the lower range of frequencies $20-800 \mathrm{~Hz}$. The biggest difference between calculated and referential results can also be seen in a higher frequency range of 500-5000 Hz.

The proposed analytical approach, which treats the test subject as an infinite plate, allows a rough estimation of noise generated by vibrating the beam excited by a hammer and modal shaker. For better estimation of SPLs, a correlation factor was proposed, which lead to the improvement of the overall estimation of SPL in the lower frequencies, even up to $1000 \mathrm{~Hz}$. The difference in SPL's absolute values is different due to the nature of the excitation source, and the duration and location of the signal, but it allows noticing the similarities in dynamic behaviour of the beam, even with different types of excitation. FEM model should be created to analyse the problem more thoroughly and get better estimation compatibility.

In further work, it is planned to focus on different analytical method for noise prediction, for which also the creation of an FEM model will be needed. The expansion of the research data with outdoor measurements and different types of excitation with force reference measurement for model improvement should also be considered. 


\section{References}

[1] Bień J., Uszkodzenia i diagnostyka obiektów mostowych. Wydawnictwa Komunikacji i Łączności, Warszawa 2010, pp. 13-24.

[2] Liang L., Li X., Zheng J., Lei K., Gou H., "Structure-borne noise from lon-span steel truss cablestayed bridge under damping pad floating slab: Experimental and numerical analysis", Applied Acoustics, vol. 157, 2020. https://doi.org/10.1016/j.apacoust.2019.07.036

[3] Okuzono T., Otsuru T., Tomiku R., Okamoto N., "A finite-element method using dispersion reduced spline elements for room acoustics simulation", Applied Acoustics, vol. 79, 2014, pp. 1-8. https:// doi.org/https://doi.org/10.1016/j.apacoust.2013.12.010

[4] Swapnil L.S., Mahesh M.S., Shakti S.G., Shantanu V.K., "Vibroacoustic study of a point-constrained plate mounted in a duct", Journal of sound and vibration, vol. 422, 2018, pp.204-226. https://doi. org/10.1016/j.jsv.2018.01.043

[5] Zhang X., Li X., Liu Q., "Theoretical and experimental investigation on bridge-borne noise under moving high-speed train", Science China Technological Sciences, vol. 56, 2013, pp. 917-924. https://doi.org/10.1007/s11431-013-5146-0

[6] Yin J., Hopkins C., "Treating periodic ribbed plates with symmetric ribs as individual subsystems in Statistical Energy Analysis: Models for bending wave transmission across L-junctions in the low- and mid-frequency ranges", Journal of sound and vibration, vol. 344, 2015, pp. 221-241. https://doi.org/10.1016/j.jsv.2015.01.031

[7] Liang L. et al., "Structure-borne noise from lon-span steel truss cable-stayed bridge under damping pad floating slab: Experimental and numerical analysis", Applied Acoustics, vol. 157, 2020, 106988. https://doi.org/10.1016/j.apacoust.2019.07.036

[8] Li Q., Xu Y.L., Wu D.J., “Concrete bridge-borne low frequency noise simulation based on traintrack-bridge dynamic interaction", Journal of sound and vibration, vol. 331, 2012, pp. 2457-2470. https://doi.org/10.1016/j.jsv.2011.12.031

[9] Xiaozhen L., Quanmin L., Shiling P., Lizhong S., Xun Z., "Structureborne noise of railway composite bridge. Numerical simulation and experimental validation", Journal of sound and vibration, vol. 353, 2015, pp. 378-394. https://doi.org/10.1016/j.jsv.2015.05.030

[10] Sestieri A., Carcaterra A., "Vibroacoustic: The challenges of a mission impossible?", Mechanical Systems and Signal Processing, vol. 34, 2013, pp. 1-18. https://doi.org/10.1016/j.ymssp.2012.08.010

[11] Cremer L., Heckl M., Ungar E., Structure-Borne Sound. Springer-Verlag, Berlin 1988, pp. 491-564.

[12] Zieliński T., Cyfrowe przetwarzanie sygnałów, Od teorii do zastosowań. Wydawnictwa Komunikacji i Łączności, Warszawa, 2005. 